\title{
Stellar to Substellar Model Atmospheres
}

\author{
France Allard, Derek Homeier, and Bernd Freytag \\ Centre de Recherche Astrophysique de Lyon, \\ UMR 5574, CNRS, Université de Lyon, \\ École Normale Supérieure de Lyon, \\ 46 Allée d'Italie, F-69364 Lyon Cedex 07, France, \\ email: france.allard@ens-lyon.fr
}

\begin{abstract}
The spectral transition from Very Low Mass stars (VLMs) to brown dwarfs (BDs) and planetary mass objects (Planemos) requires model atmospheres that can treat line, molecule, and dust-cloud formation with completeness and accuracy. One of the essential problems is the determination of the surface velocity field throughout the main sequence down to the BD and planemo mass regimes. We present local $2 \mathrm{D}$ and $3 \mathrm{D}$ radiation hydrodynamic simulations using the CO5BOLD code with binned Phoenix gas opacities, forsterite dust formation (and opacities) and rotation. The resulting velocity field vs depth and Teff has been used in the general purpose model atmosphere code Phoenix, adapted in static 1D spherical symmetry for these cool atmospheres. The result is a better understanding of the spectral transition from the stellar to substellar regimes. However, problems remain in reproducing the colors of the dustiest brown dwarfs. The global properties of rotation can change the averaged spectral properties of these objects. Our project for the period 2011-2015 is therefore to develop scaled down global 3D simulations of convection, cloud formation and rotation thanks to funding by the Agence Nationale de la Recherche in France.
\end{abstract}

Keywords. stars, brown dwarfs, planets, model atmospheres, hydrodynamic simulations.

\section{Introduction}

Since infrared observations of M dwarf stars (late 80s), brown dwarfs (mid 90s), and extrasolar planets (mid 2000s) became available, one of the most important challenges in modeling their atmospheres has become the understanding of molecular opacities at high temperatures (where the high energy transitions can only be captured by ab initio calculations) and dust cloud formation. Their compositions, assumed solar in general, take the form of molecular hydrogen, $\mathrm{N}_{2}$ and $\mathrm{CO}$, while the leading opacities in their spectra is due to titanium oxide and water vapor bands, with smaller contributions from vanadium oxide and various hydrides in the optical spectra range, and CO bands in the infrared. Model atmospheres and synthetic spectra have been improving along with the quality of these molecular opacities. The treatment of water vapor alone - which begins to form in early type $\mathrm{M}$ dwarfs (around Teff $=3500 \mathrm{~K}$ ) and is present in Very Low Mass stars (VLMs), brown dwarfs, and planets including Hot Jupiters reaching an equilibrium temperature with the star of around $1600 \mathrm{~K}$ - has seen an important evolution throughout the years: from band model approximations, to straight means based on the hot flames experiments (Ludwig 1971) used in our early model atmosphere grids (Allard 1990, Allard \& Hauschildt 1995), to ab initio computations by Partridge \& Schwenke (1997) (see also Allard, Hauschildt \& Schwenke 2000), and to the current ab initio line lists by Baber et al. (2006) used in the most recent models. Nevertheless, the models atmosphere have systematically failed to reproduce the relative strength and shapes of the water bands, as shown for the case of an M dwarf in Fig. 1. 


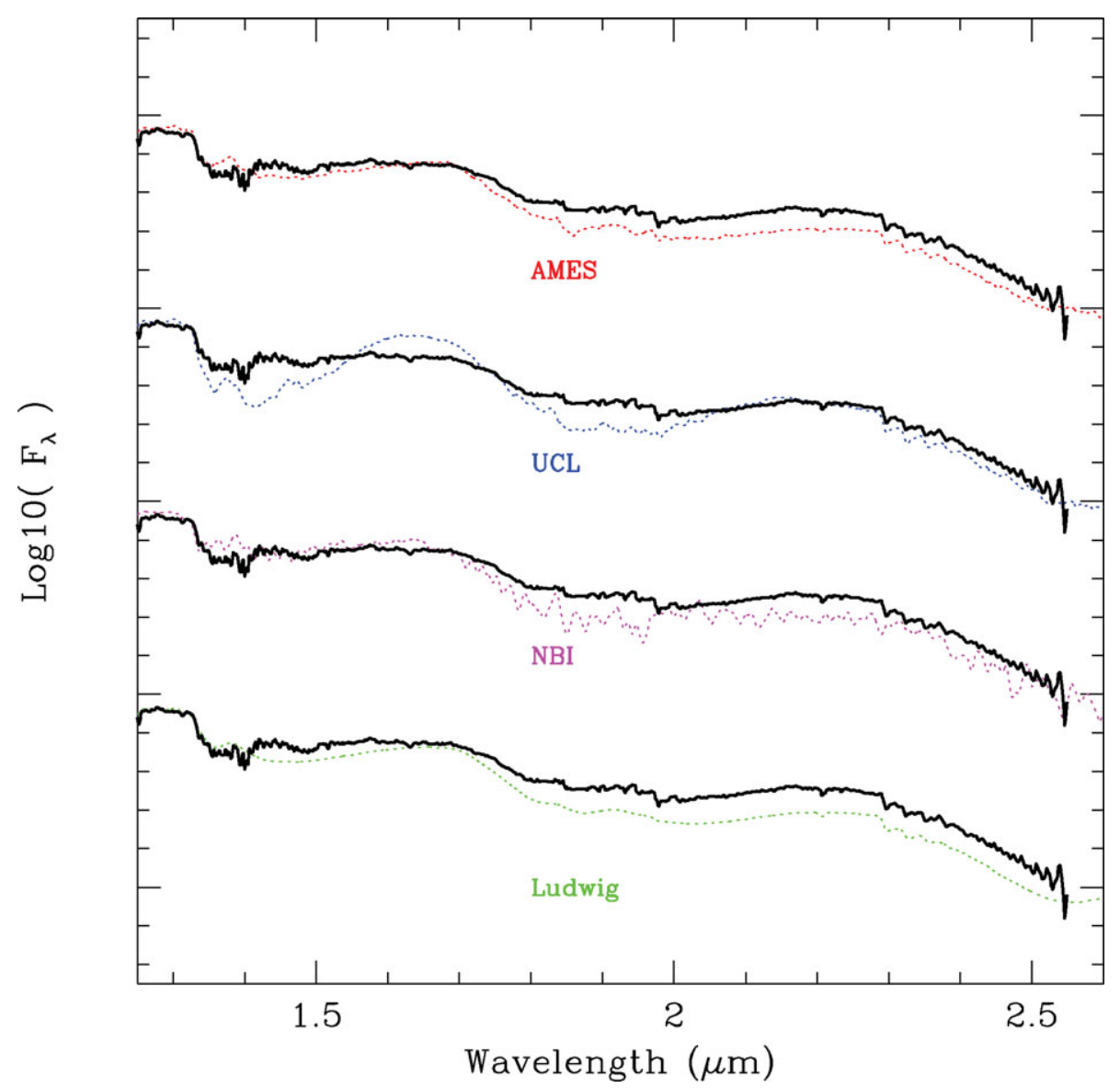

Figure 1. The infrared SED of the M8e dwarf star VB10 (thick full line) is compared to Phoenix synthetic spectra using different water vapor opacity profiles (dotted lines) from bottom to top: the hot flames experiments by Ludwig (1971), labeled as Ludwig, used in the early model grids for VLMs by Allard (1990) and Allard \& Hauschildt (1995); the ab initio though incomplete line list by Schryber, Miller, \& Tennyson (1995), labeled as UCL, used in the revised opacity-sampling NextGen model grid for VLMs by Hauschildt et al. (1999a,b), and the very complete ab initio line list by Partridge \& Schwenke (1997), labeled as AMES, used in the limiting case for dust formation AMES-Cond/Dusty model grids for brown dwarfs by Allard et al. (2001). Results obtained for the line list by Jørgensen et al. (2001), labeled NBI, is also shown for comparison.

\section{The impact of revised solar abundances on VLMs properties}

The modeling of atmospheres has also evolved with the development of computing capacities from an analytical treatment of the transfer equation using moments of the radiation field (e.g. Allard, 1990), to a line-by-line opacity sampling in spherical symmetry (e.g. Hauschildt et al. 1999a,b), and to 3D radiation transfer (e.g. Seelmann, Hauschildt \& Baron 2010). In parallel to detailed radiative transfer in an assumed static environment, hydrodynamical simulations have been developed to reach a realistic representation of the granulation and the line profiles shifted and shaped by the hydrodynamical flow of the Sun and Sun-like stars (see a review by Freytag et al. in a special issue of the Journal of Computational Physics to appear) by using a non-grey (multi-group opacities) radiative 


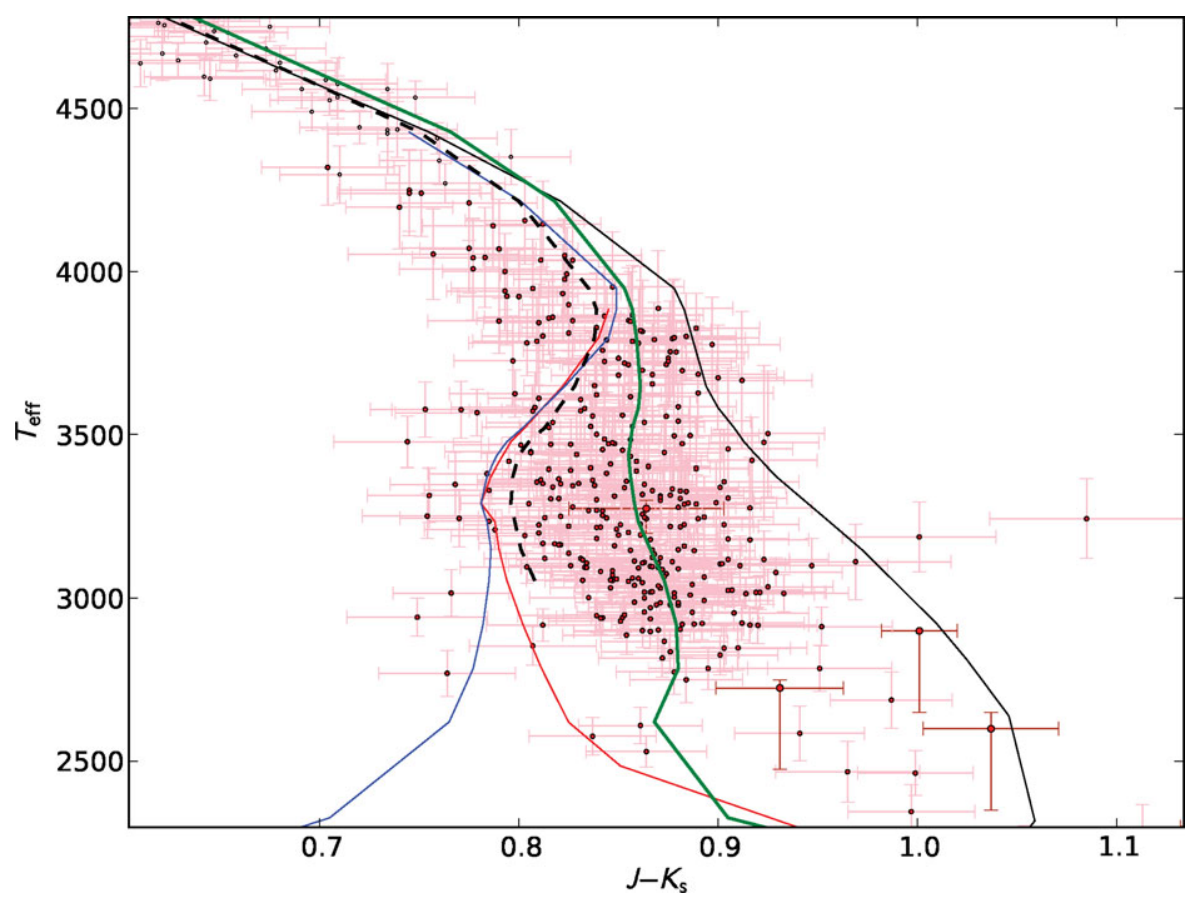

Figure 2. Estimated Teff for M dwarfs by Casagrande, Flynn, \& Bessell (2008) (dots with grey error bars) and brown dwarfs by Golimowski et al. (2004) (dots with black error bars) are reported as a function of J-Ks . Over-plotted are the NextGen model isochrones for 5 Gyrs (Baraffe et al. 1998) using various generations of model atmospheres for the interpolation into the observational plane, starting with the NextGen (full line redwards of the bulk of stars), the limiting case AMES-Cond/Dusty grids by Allard et al. (2001) (full lines bluewards of the bulk of $\mathrm{M}$ dwarfs and splitting below $3300 \mathrm{~K}$ bluewards and redwards respectively), and with the BT-Settl models using the Asplund et al. (2009) solar abundances (full line running through the bulk of M dwarfs). A version of the BT-Settl models, called BT-NextGen, using the Grevesse, Noels \& Sauvals (1993) abundances are shown (dashed line) to illustrate the effects of the revised solar abundances.

transfer with a blackbody source function (scattering is neglected). Using such local 3D simulations and revised signal to noise and spectrally resolved observations, Asplund et al. (2009) and Caffau et al. (2011) have been able to redefine the solar abundances. These two independent groups agree in favoring a reduced (by a factor 2) solar oxygen abundance compared to previous values (Grevesse, Noels \& Sauval 1993).

The revised lower oxygen abundances help models to reproduce the spectral properties of M dwarfs compared to earlier models based on previous estimates of the solar abundances by Grevesse, Noels, \& Sauval (1993). This can be seen from Fig.2, where we compare models to the empirical determinations of Teff of individual stars by Golimowski et al. (2008). The J bandpass corresponds to the peak of the Spectral Energy Distribution (or SED) of $\mathrm{M}$ dwarfs, and is relatively insensitive to changes in atmospheric composition; while the Ks bandpass is very sensitive, with a broad sodium doublet, $\mathrm{H}_{2}$ CollisionInduced Absorption (or CIA), CO bands in M dwarfs, and methane bands in late-type brown dwarfs. We compare the published NextGen and AMES-Cond/Dusty models, as well as the new calculations: i) the new BT-Settl models using the Asplund et al. (2009) solar abundances, ii) the new BT-NextGen models using solar abundances by Grevesse, Noels, \& Sauval (1993) to allow isolating the effect of the revised oxygen abundance, and 
iii) the new BT-Cond/Dusty models corresponding to the published AMES-Cond/Dusty models in terms of the treatment of the limiting effects of dust formation to isolate the effects of the revised Phoenix code version. All the models use the same published interior and evolution models: i) the NextGen isochrones of Baraffe et al. (1998) in the stellar regime, ii) the AMES-Dusty isochrones of Chabrier et al. (2000) for the transition regime between $\mathrm{M}$ dwarfs and brown dwarfs, and iii) the AMES-Cond isochrones of Baraffe et al. (2003) in the late type brown dwarf to planetary mass regime. Evolution models are currently being prepared using the BT-Settl model atmosphere grid. One can see from this comparison that the NextGen models systematically overestimate Teff throughout the lower main sequence, while the AMES-Cond/Dusty models slightly underestimate Teff. This situation is relieved when using the revised solar abundances, and the BT-Settl models now agree fairly well with most of the empirical estimations of Teff. The scatter in this diagram is due to the presence of dwarfs from several populations (metallicity), and an improvement would be to identify these populations.

We also find (not shown $\dagger$ ) that the BT-Settl models reproduce also satisfactorily the complete SED of M dwarfs, solving a major historical issue as can be seen from Fig.1 (see also Allard, Hauschildt \& Schwenke 2000). Some discrepancy remains in the K band which can be due to slightly overestimated $\mathrm{H}_{2}$ CIA opacities. The agreement is also excellent in the optical to red part of the spectrum, in particular in the FeH Wing Ford bands near $0.99 \mu \mathrm{m}$, and in the VO bands thanks to line lists provided by B. Plez (GRAAL). Missing opacities are, however, still affecting the spectral distribution $(\mathrm{CaOH}$ bands), and current titanium oxide line lists become now too incomplete and inaccurate compared to the precision of the current water vapor opacities. Revised opacities are expected from the UCL group thanks to an ERC grant to J. Tennyson (UCL) for the computation of ab initio molecular opacities.

\section{Dust formation in late type VLMs and brown dwarf atmospheres}

An important additional difficulty has been identified in the greenhouse or backwarming effects due to dust cloud formation in late-type $M$ dwarf and brown dwarf atmospheres by Tsuji (1996a,b). Oxygen-rich dust grains (silicates) deplete as much as $20 \%$ of the oxygen content of the gas, increasing the apparent $\mathrm{C} / \mathrm{O}$ ratio. The formation of dust clouds and its associated greenhouse effect cause the infrared colors of late $\mathrm{M}$ and early L dwarfs to become extremely red compared to the colors of low-mass stars. The cloud composition, according to equilibrium chemistry, is going from zirconium oxide $\left(\mathrm{ZrO}_{2}\right)$, to refractory ceramics (perovskite and corundum; $\mathrm{CaTiO}_{3}, \mathrm{Al}_{2} \mathrm{O}_{3}$ ), to silicates (e.g. forsterite; $\mathrm{Mg}_{2} \mathrm{SiO}_{4}$ ), to salts ( $\mathrm{CsCl}, \mathrm{RbCl}, \mathrm{NaCl}$ ), and finally to ices $\left(\mathrm{H}_{2} \mathrm{O}, \mathrm{NH}_{3}\right.$, $\mathrm{NH}_{4} \mathrm{SH}$ ), as brown dwarfs cool down with time from $\mathrm{M}$ through $\mathrm{L}$, and $\mathrm{T}$ spectral types and beyond (Allard et al. 2001; Lodders \& Fergley 2006). Many cloud models have been constructed to address this problem in brown dwarfs over the past decade (see the review by Helling et al. 2008 for a comparison of the various models). However, none treated the mixing properties of the atmosphere and the resulting diffusion mechanism realistically enough to reproduce the properties of the spectral transition from $M$ through $\mathrm{L}$ and $\mathrm{T}$ spectral types without changing cloud parameters (e.g. Ackerman \& Marley 2001). It is in this context that we have decided to address the issue of mixing and diffusion by $2 \mathrm{D}$ radiation hydrodymanic simulations of VLMs and brown dwarfs atmospheres (Teff=2800K to $900 \mathrm{~K}$ ), using the Phoenix gas opacities in a multi-group, a two

$\dagger$ These results will be demonstrated at the occasion of the detailed publication of the final BT-Settl model grid. 


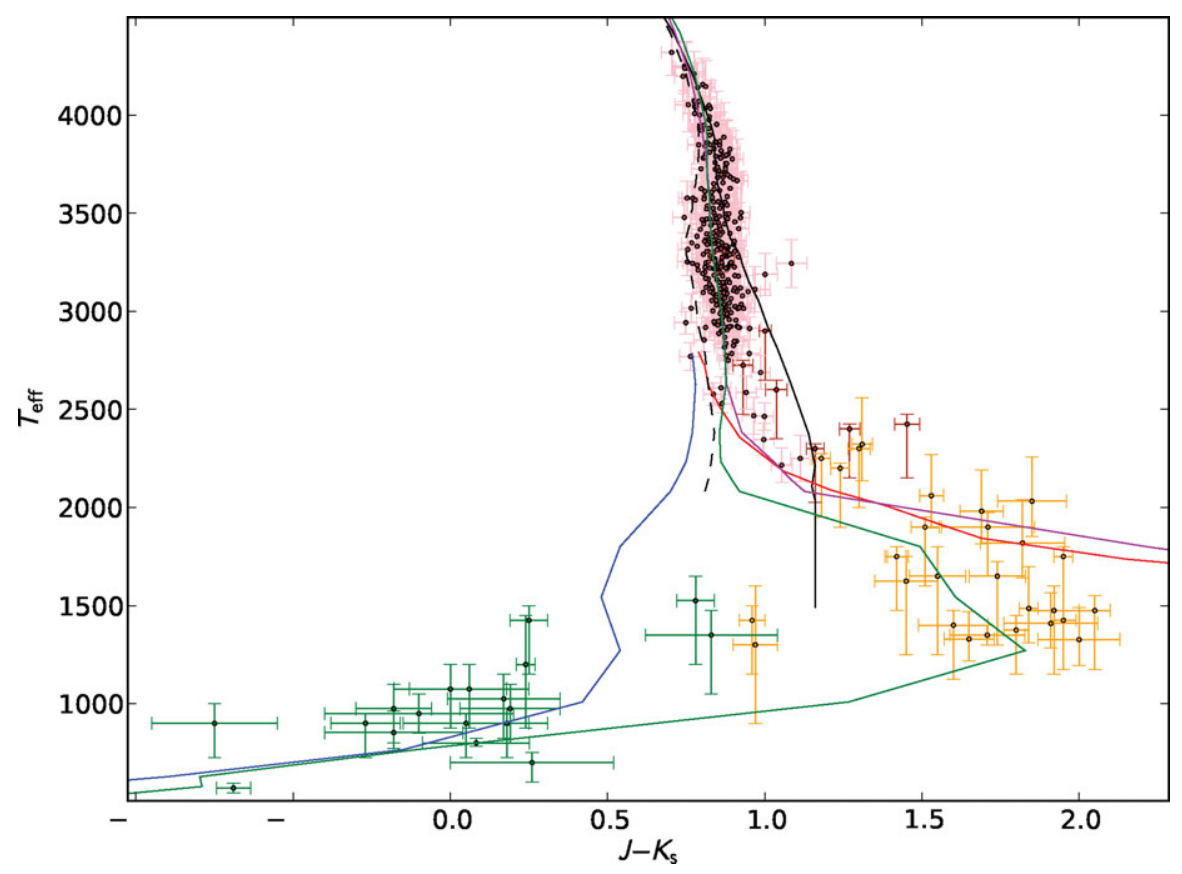

Figure 3. Same plot as Fig.2 but zooming out and extending into the brown dwarf region of the diagram. The $\mathrm{J}$ band corresponds to the peak of the SED while the Ks band is sensitive to the composition of the atmosphere (see text). The region between $2500 \mathrm{~K}$ and $1400 \mathrm{~K}$ is dominated by dust formation (essentially forsterite and other silicates) and $\mathrm{CO}$ absorption in the $\mathrm{K}$ band, while cooler brown dwarfs show methane formation and a turn to the blue of the J-Ks color. The limiting case AMES-Cond/Dusty models atmosphere provide a description of the span in colors of the brown dwarfs in this diagram. We use isochrones of 5 Gyrs for the purpose of illustration.

bin (monomers and dust) cloud model and forsterite geometric cross-sections (Freytag et al. 2010). We discovered the formation of gravity waves as one driving mechanism for the formation of clouds in these atmospheres. Around Teff $\lesssim 2200 \mathrm{~K}$, the cloud layers become optically thick enough to initiate cloud convection, which participate in the global mixing. Overshoot is also important in the mixing of the largest dust particles.

We derived a rule for the velocity field versus atmospheric depth and Teff, which is relatively insensitive to gravity. This rule was then used in the construction of the new model atmosphere grid BT-Settl. The cloud model used in the BT-Settl models is based on the condensation and sedimentation timescales from a study of planetary atmospheres by Rossow (1978). However, one improvement compared to previous model versions (see e.g. Helling et al. 2008), is that we compute supersaturation from our pre-tabulated equilibrium chemistry in order to obtain the correct amount of dust formation (as opposed to the value suggested by Rossow). We solve the cloud model and equilibrium chemistry layer by layer outward to account for the history of grain formation as a function of the cooling of the gas. One can see from Fig. 3 that the late-type $\mathrm{M}$ and early type $\mathrm{L}$ dwarfs behave as if dust is formed nearly in equilibrium with the gas phase with extremely red colors, in good agreement with the Dusty models. At the low Teff regime dominated by T-type dwarfs, the AMES-Cond models also appear to provide a good limitation for late-type brown dwarfs. The improved BT-Settl cloud model shows promising results in describing the stellar to substellar transition. It appears, however, that not enough dust 

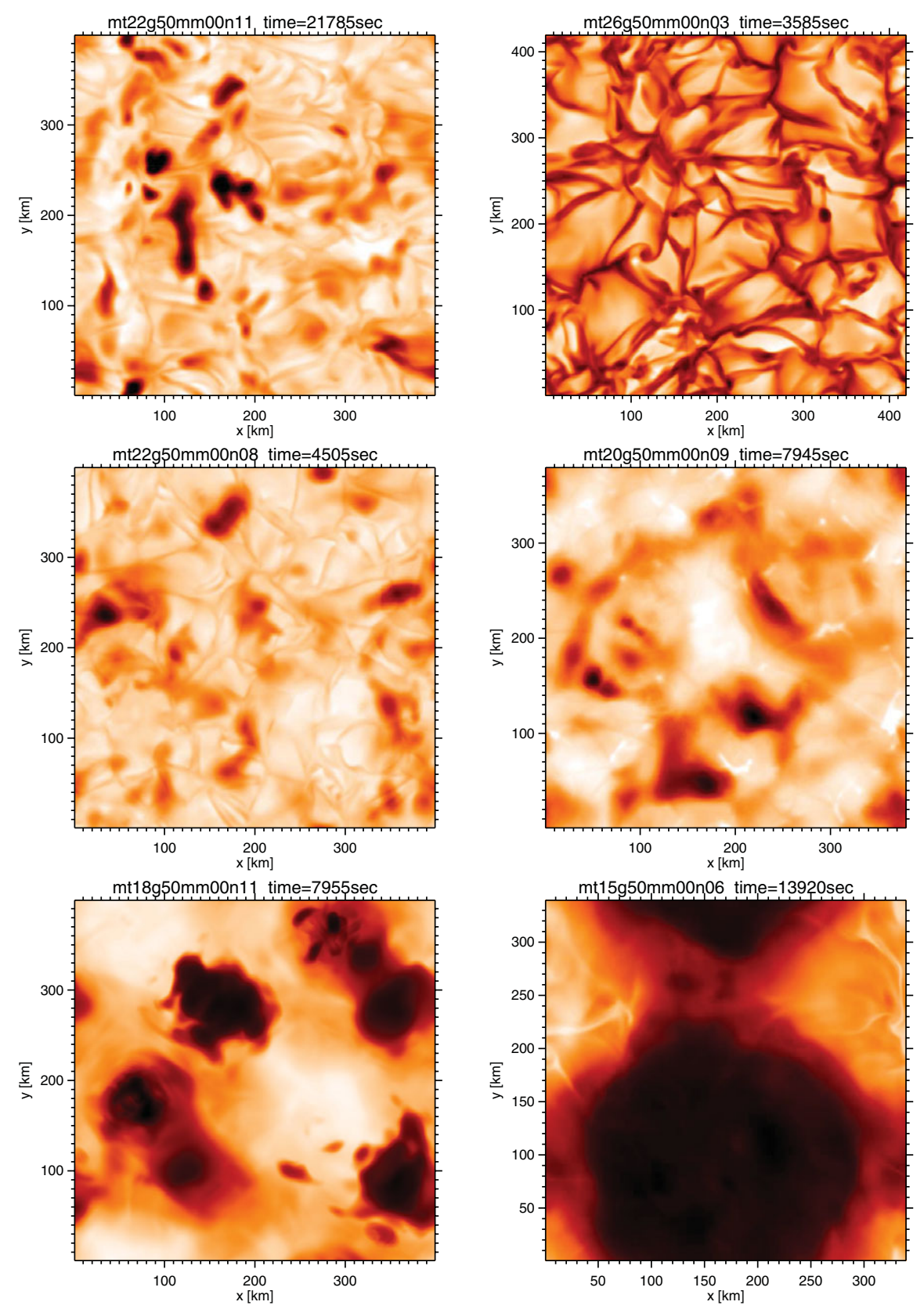

Figure 4. Snapshots of the intensity of local 3D RHD simulations at the surface of, from top to right to bottom right, $2600 \mathrm{~K}, 2200 \mathrm{~K}, 2000 \mathrm{~K}, 1800 \mathrm{~K}$ and $1500 \mathrm{~K}$ atmospheres of $\operatorname{logg}=5.0$, solar metallicity and no rotation, except for the $2200 \mathrm{~K}$ case shown (top left) using a rotational period of 15 minutes to highlight the effects on the convection cells and the cloud formation.

is predicted at the lower gravities predicted by evolution models for 5 Gyrs $(\log g=4.0$ to 3.5) to reproduce the reddest brown dwarfs with these isochrones. Revised interior and evolution models using the new solar abundances and the BT-Settl atmospheres as surface boundary are expected by mid-2012. 
While the distribution of dust with depth is important to understand the SED and possible spectral variability of VLMs, brown dwarfs and planets, the surface distribution of dust also matters. For this, 3D global or "star in the box" RHD simulations with rotation are required. This is our current project supported by the French "Agence Nationale de la Recherche" for the period 2010-2015. Rotation is already included in CO5BOLD for a scaled down model of the Sun (Steffen \& Freytag (2007) and can be applied to brown dwarf and planet simulations. In Fig.4, 3D RHD simulation boxes at the surface of the star) are shown for dwarfs with Teff ranging across the stellar to brown dwarfs transition. The $2600 \mathrm{~K}$ case shows no or negligible dust formation, while dust formation progresses to reach optically thick density at around $2200 \mathrm{~K}$, before sedimenting out again towards the $1300 \mathrm{~K}$ regime (not shown). The Teff $=1500 \mathrm{~K}$ case illustrates the importance of gravity waves, where the minima of the waves reach condensation levels while the maxima remain in condensed phase. Also shown is the case of a $2200 \mathrm{~K}$ simulation using a wind at the equator corresponding to a 15 mins period rotation of the star). This period of rotation is very extreme compared to the averaged period of rotation of brown dwarfs ( $2 \mathrm{hrs}$ ), and illustrates the stability of the dust formation process against rotation. The simulation boxes are, however, too small compared to the stellar radius to show the overall variability. For this purpose, scaled down global 3D simulations of red dwarfs, brown dwarfs, and planets are currently developed using CO5BOLD.

\section{Summary}

We present in this paper, a brief overview of the main preliminary highlights of the BTSettl model atmosphere grid for stars, brown dwarfs, and young planets computed using the atmosphere code Phoenix, which has been updated since Allard et al. (2001), with: i) spherical symmetry and non-LTE for most elements, ii) alpha element enrichment, iii) the Barber et al. (2006) BT2 water opacity line list, iv) solar abundances revised by Asplund et al. (2009), and v) a cloud model accounting for an improved treatment of supersaturation and RHD mixing. These models will be published in greater detail shortly in A\&A. The grid spans $100,000 \mathrm{~K}<T_{\text {eff }}<400 \mathrm{~K} ;-0.5<\operatorname{logg}<5.5$; and $+0.5<[\mathrm{M} / \mathrm{H}]$ $<-1.5$. The models are available at the Phoenix simulator website "http://phoenix.enslyon.fr/simulator/" and are in preparation for publication. They will serve the GAIA, MUSE, and PLATO missions for which a detailed comparison of models from various authors is planned.

We find that the BT-Settl models with reduced oxygen abundance show a significant improvement in reproducing $\mathrm{M}$ dwarfs, while the improved cloud model allows an improved reproduction of the turn to the red due to dust formation before predicting a turn over to blue below $1700 \mathrm{~K}$ in J-Ks colors. The models, however, appear not to be forming enough high altitude dust to reproduce the most extreme dusty brown dwarfs in this diagram. Improvements to our computed supersaturation and porosity of grains are possible venues of refinement. The RHD simulations of Freytag et al. (2010) have shed light on the importance of mixing in the atmospheres of VLMs, brown dwarfs, and possibly planets. These results imply that the gas is out of equilibrium. Beyond molecular opacities, model atmospheres require, therefore, reaction rates for the most abundant molecules and most important absorbers.

To address the new source of spectroscopic information on Hot Jupiters and young imaged planets to come, we have developed the BT-Settl model atmosphere grid to encompass the parameter regime of these objects (low gravity around $\log g=4.0$, Teff below $2000 \mathrm{~K}$ ). These models are those of single objects, but the development of the 
binary star function of the Phoenix simulator will shortly allow the study of impinging irradiation effects on planetary atmospheres.

\section{Acknowledgements}

We would like to thank the French "Agence Nationale de la Recherche" (ANR) and "Programme National de Physique Stellaire" (PNPS) of CNRS (INSU) for their financial support. The computations of dusty $\mathrm{M}$ dwarf and brown dwarf models were performed at the Pôle Scientifique de Modélisation Numérique (PSMN) at the École Normale Supérieure (ENS) in Lyon.

\section{References}

Ackerman, A. S. \& Marley, M. S. 2001, ApJ, 556, 872

Allard, F. 1990, Ph.D. thesis, PhD thesis. Ruprecht Karls Univ. Heidelberg, (1990)

Allard, F. \& Hauschildt, P. H. 1995, ApJ, 445, 433

Allard, F., Hauschildt, P. H., Alexander, D. R., Tamanai, A., \& Schweitzer, A. 2001, ApJ, 556, 357

Allard, F., Hauschildt, P. H., \& Schwenke, D. 2000, ApJ, 540, 1005

Asplund, M., Grevesse, N., Sauval, A. J., \& Scott, P. 2009, ARAA, 47, 481

Baraffe, I., Chabrier, G., Allard, F., \& Hauschildt, P. H. 1997, A\& A, 327, 1054

- 1998, A\& A, 337, 403

Baraffe, I., Chabrier, G., Barman, T. S., Allard, F., \& Hauschildt, P. H. 2003, A\&A, 402, 701

Barber, R. J., Tennyson, J., Harris, G. J., \& Tolchenov, R. N. 2006, MNRAS, 368, 1087

Caffau, E., Ludwig, H.-G., Steffen, M., Freytag, B., \& Bonifacio, P. 2011, Solar Phys., 268, 255

Casagrande, L., Flynn, C., \& Bessell, M. 2008, MNRAS, 389, 585

Chabrier, G., Baraffe, I., Allard, F., \& Hauschildt, P. 2000, ApJ, 542, 464

Freytag, B., Allard, F., Ludwig, H., Homeier, D., \& Steffen, M. 2010, A\&A, 513, A19

Golimowski, D. A. \& collaborators, 2004, AJ, 127, 3516

Grevesse, N., Noels, A., \& Sauval, A. J. 1993, A\&GA, 271, 587

Hauschildt, P. H., Allard, F., \& Baron, E. 1999a, ApJ, 512, 377

Hauschildt, P. H., Allard, F., Ferguson, J., Baron, E., \& Alexander, D. R. 1999b, ApJ, 525, 871

Helling, C., Ackerman, A., Allard, F., Dehn, M., Hauschildt, P., Homeier, D., Lodders, K., Marley, M., Rietmeijer, F., Tsuji, T., \& Woitke, P. 2008, MNRAS, 391, 1854

Jørgensen, U. G., Jensen, P., Sørensen, G. O., \& Aringer, B. 2001, A\& $A$, 372, 249

Lodders, K. \& Fegley, Jr. B. 2006, In Astrophysics Update 2, editor: Mason J. W. Spinger Verlag, p1.

Ludwig, C. B. 1971, Applied Optics, 10, 1057

Partridge, H. \& Schwenke, D. W. 1997, Journal for Computational Physics, 106, 4618

Rossow, W. B. 1978, ICARUS, 36, 1

Schryber, J. H., Miller, S., \& Tennyson, J. 1995, JQSRT, 53, 373

Seelmann, A. M., Hauschildt, P. H., \& Baron, E. 2010, A\& A, 522, A102

Steffen, M. \& Freytag, B. 2007, AN, 328, 1054

Tsuji, T., Ohnaka, K., \& Aoki, W. 1996a, A\&A, 305, L1

Tsuji, T., Ohnaka, K., Aoki, W., \& Nakajima, T. 1996b, A\&3A, 308, L29

\section{Discussion}

W. KLEY: Why do you have to use a very small radius in global convection models for dwarf stars?

F. Allard: Because we need to resolve the convective cells which are so much smaller compared to the radius of the star. 